\title{
A Case of Fungal Ball with Cholesterol Granuloma in the Middle Ear Cavity
}

\author{
Su Geun Kim ${ }^{1,2}$, Eun Jung Lee ${ }^{1,2}$, Ji Seob Yoo ${ }^{1,2}$, and Cha Dong Yeo ${ }^{1,2}$ \\ ${ }^{1}$ Department of Otorhinolaryngology Head and Neck Surgery, Chonbuk National University College of Medicine, Jeonju, Korea \\ ${ }^{2}$ Research Institute of Clinical Medicine of Chonbuk National University-Biomedical Research Institute, \\ Chonbuk National University Hospital, Jeonju, Korea
}

\author{
Received August 28, 2019 \\ Revised September 27, 2019 \\ Accepted October 24, 2019

Address for correspondence
Eun Jung Lee, MD
Department of Otorhinolaryngology
Head and Neck Surgery,
Chonbuk National University
College of Medicine,
20 Geonji-ro Deokjin-gu,
Jeonju 54907, Korea
Tel +82-63-250-1980
Fax+82-63-250-1986
E-mail imaima97@naver.com

Fungal balls consist of rounded conglomerates of fungal mycelia, which can form within a preexisting cavity. They are mostly found in the paranasal sinuses in the head and neck regions. Cholesterol granuloma is a fibrotic lesion that develops as a tissue response to a foreign body such as cholesterol crystals or hemosiderin and is often associated with chronic otitis media. We present the unusual case of a 62-year-old male who was treated for chronic otitis media, which was histologically confirmed as a fungal ball and cholesterol granuloma in the middle ear cavity following tympanomastoidectomy. This is the first reported case of synchronous fungal ball and cholesterol granuloma in the middle ear cavity. J Audiol Otol 2020;24(4):210-213

KEY WORDS: Fungal ball $\cdot$ Cholesterol granuloma $\cdot$ Middle ear.

\section{Introduction}

Fungal balls are infections characterized by aggregates of fungal mycelia that colonize a preexisting cavity. It is mostly found in the paranasal sinuses in the head and neck regions. The most commonly involved site is maxillary sinus. Although the pathogenesis is still unclear, previous studies indicate the role of sex and age as fungus ball is predominant in females and those of older age. Cholesterol granuloma (CG) is a fibrotic lesion that develops as a tissue response to a foreign body such as cholesterol crystals or hemosiderin and is often associated with chronic otitis media. These lesions can be found in the middle ear cavity, mastoid air cells, and petrous apex. Most of these are asymptomatic and do not require treatment. Surgical management is necessary if it involves adjacent structures such as the inner ear, internal auditory canal, cranial nerves, or if when they are painful. Cholesterol granulomas sometimes look like benign masses. There are many case reports from the

This is an Open Access article distributed under the terms of the Creative Commons Attribution Non-Commercial License (https://creativecommons.org/licenses/by-nc/4.0/) which permits unrestricted non-commercial use, distribution, and reproduction in any medium, provided the original work is properly cited. literature, many of which have been clinically suspected of benign tumors but diagnosed with $\mathrm{CG}$. We present a rare case of a 62-year-old male who diagnosed with chronic otitis media, which was histologically confirmed as fungal ball and CG in the middle ear cavity following ear surgery. To the best of our knowledge, there have been no reports of synchronous fungal ball and CG in the middle ear cavity.

\section{Case Report}

A 62-year-old farmer living in a rural area presented to our department with complaints of right-sided otorrhea, hearing impairment, and tinnitus for 2 months. He had previously been treated with topical and systemic antibiotics at another clinic. The patient denied dizziness and other vestibular symptoms. He reported the use of inhaled corticosteroids for chronic obstructive pulmonary disease and had no history of ear infections, previous ear surgery, or any other illness. Otoscopic examination of the right ear showed near-total perforation of the tympanic membrane with a purulent discharge. The eardrum was severely inflamed with a white mass-like lesion in the center. There was no clinical suspicion of otomycosis as the findings 
did not reveal the presence of fungal hyphae. The mass of tissue located in the promontory area was soft to touch. It was provisionally diagnosed as a benign soft tissue tumor or $\mathrm{CG}$ (Fig. 1A). The external auditory canal (EAC) was also severely inflamed. Otoscopy of the left ear revealed an intact tympanic membrane and EAC. Audiologic evaluation showed conductive hearing loss in the right ear (Fig. 2A). A high-resolution computed tomography (CT) scan of the temporal bone revealed soft tissue density in the middle ear cavity and mastoid cells, indicating chronic otitis media (Fig. 3).

We cultured the ear discharge, initiated topical and systemic antibiotics for 4 months, and conducted the ear dressing regularly. However, the patient's symptoms showed a relapsingremitting course. Therefore, we decided to proceed with surgery and performed a tympanomastoidectomy. Intraoperative findings showed cheese-like, necrotic granulation tissue originat-
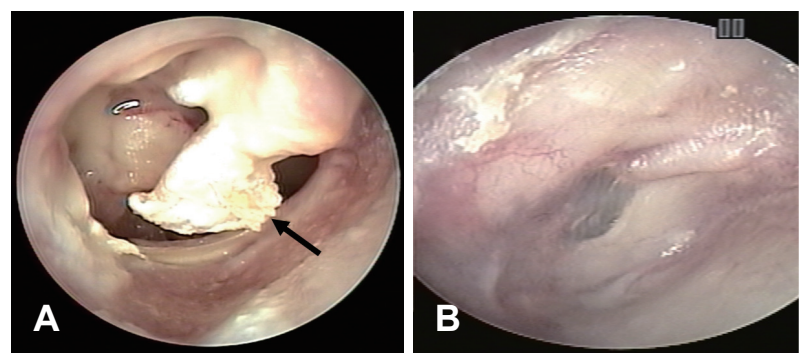

Fig. 1. Preoperative view of otoscopy. (A) Preoperative view of the right ear showing near-total perforation of the eardrum with purulent discharge and with a whitish mass-like lesion in the center (arrow). The mass-like lesion originated in the promontory and attached to the malleus handle. The eardrum is also seen to be severely swollen. (B) Postoperative view showing a slightly pale but intact eardrum without otorrhea.

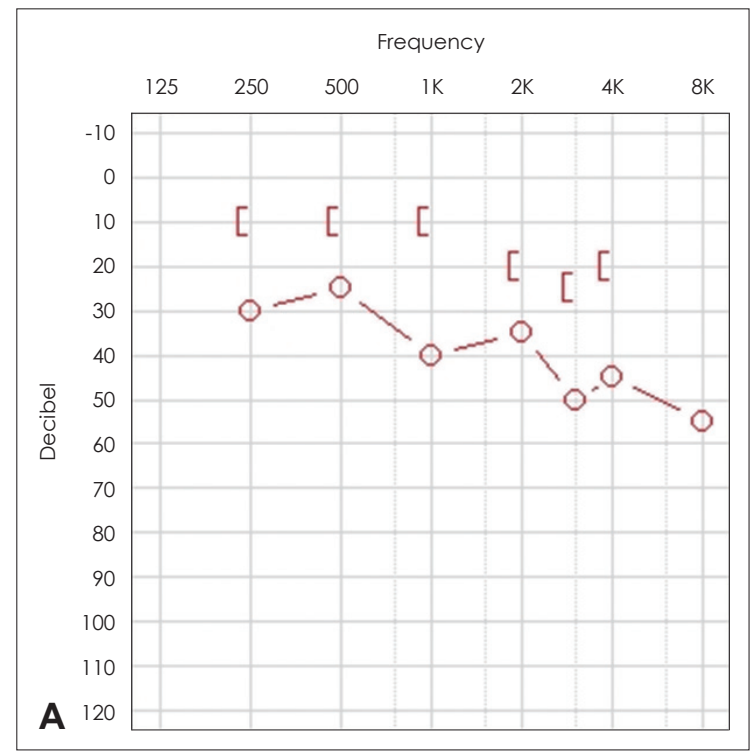

ing from the promontory area and another area of granulation tissue surrounding the incus and incudostapedial joint, which extended to the attic. Ossicular status and mobility were normal, and the pharyngotympanic tube was obstructed with granulation tissue. We performed a canal-wall-up mastoidectomy with a composite type I tympanoplasty. Histopathologic findings confirmed the diagnosis of a fungal ball (Aspergillus spp.) for the surgical specimens obtained from the promontory area and CG for the tissue near the incus (Fig. 4). Postoperatively, the patient was free of symptoms during the follow-up period of 10 months, and otoscopic examination showed a slightly pale but intact eardrum without otorrhea (Fig. 1B). Audiologic evaluation revealed a decreased air-bone gap, but the sound level was generally reduced (Fig. 2B).

\section{Discussion}

The fungus Aspergillus is a member of the phylum Thallophyta of the plant kingdom, and the term aspergillosis was first introduced by Virchow for mycosis. Fungal infections of the ear are most commonly caused by saprophytes or secondary
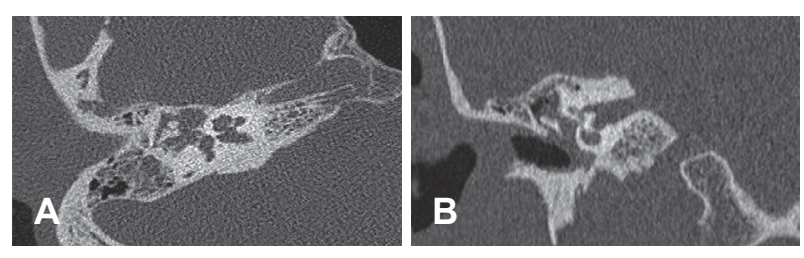

Fig. 3. Preoperative computed tomography scans. (A) Axial view. (B) Coronal view. Soft tissue density in the middle ear cavity and mastoid cells, indicating chronic otitis media.

Fig. 2. Preoperative and postoperative audiogram. (A) Preoperative audiogram showing conductive hearing loss in the right ear. (B) Postoperative audiogram showing decreased air-bone gap, but the sound level is generally reduced (Air conduction: o, Bone conduction: [, Right side masking: $\triangle$ ). K: kilohertz. 
invaders of the skin affected by eczema, psoriasis, or previous bacterial infection [1]. No cases of suppurative otitis media due to primary fungal infection have been reported in the literature where there was no disseminated fungal infection. Chronicity of the discharge is a critical determinant of fungal otitis media as humidity in the ear alters the $\mathrm{pH}$ of the media to alkaline epithelial debris [2]. Thus, fungus ball in the paranasal sinuses is a relatively common condition, often encountered in clinical settings. Fungal invasion of the middle ear cavity occurs by two routes. First, it can ascend from the nasopharynx through the pharyngotympanic tube, and the second route is through a perforated eardrum. As our patient showed no evidence of fungal infection in other sites (paranasal sinus, pharynx, larynx), the fungus likely invaded through the perforated eardrum. It has been postulated that prolonged use of topical broad-spectrum antibiotics leads to suppression of bacterial flora and the subsequent emergence of opportunistic fungi in the oral cavity, gastrointestinal tract, and vagina [3]. Similarly, prolonged use of topical antibiotics may lead to fungal infections of the middle ear [4]. Our patient had received topical antibiotics for over 4 months, raising the possibility of secondary fungal overgrowth.

Fungus balls may become invasive with deteriorating host immunity. Therefore, fungal ball formation in patients with diabetes and immunodeficiency disorders demands prompt attention and careful management. The invasive form of the dis- ease results in significant morbidity and mortality. Treatment requires surgical debridement and antifungal therapy. However, in the case of a fungal ball not invading adjacent structures or mucosa, treatment consists of complete excision combined with curettage and adequate ventilation.

CG is a histological term used to describe a tissue response to a foreign body such as cholesterol crystals or hemosiderin. Manasse first reported CG of the middle ear in 1917 [5]. The most commonly accepted theories on the pathogenesis of CG are mucosal outflow obstruction, vacuum-triggered hemorrhage, or cholesterol foreign-body reaction as a result of the anaerobic breakdown of blood products [6]. The pathogenesis of aspergilloma and CG of the middle ear is similar, as obstruction of ventilation and impaired drainage are common mechanisms between both conditions. To the best of our knowledge, the association between $\mathrm{CG}$ in the middle ear with fungal ball formation has still not been explained. So far, only two cases of simultaneously occurring CG and fungal balls have been reported, one in the maxillary sinus and the other in the sphenoid sinus [7,8]. As far as we know, ours is the first reported case involving the middle ear. A humid environment and impaired ventilation in chronic otitis media and chronic sinusitis favor the growth of CG and fungal balls. The fungal cell membrane contains ergosterol as the main component in contrast to cholesterol, which is the principal sterol present in animal cells. Therefore, the breakdown products of the fungi cannot
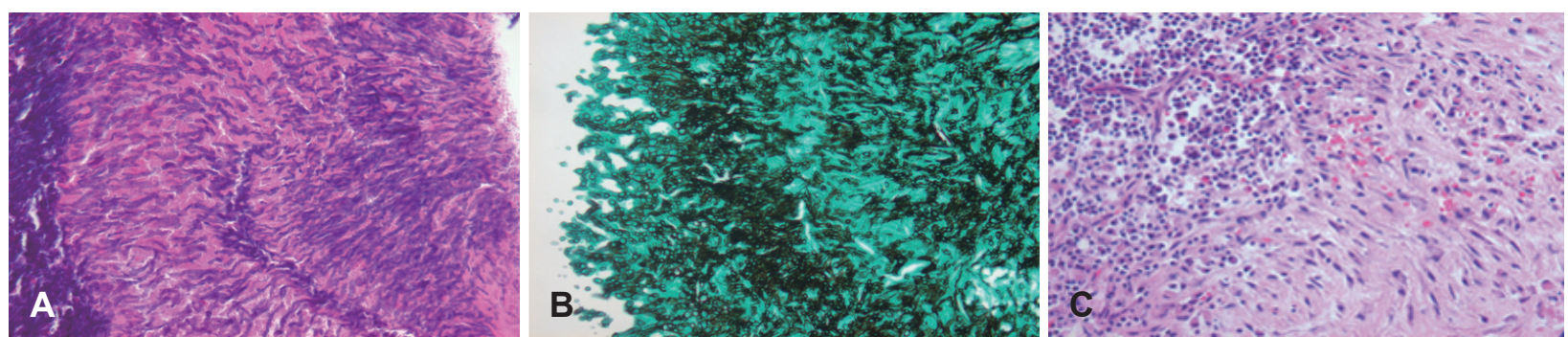

Fig. 4. Histopathological slides of specimens after surgery. (A) Tangle of branching septate hyphae with neutrophils, a finding indicative of Aspergillosis [hematoxylin and eosin (H\&E) staining, $\times 400$ ]. (B) Dichotomously branching and septating hyphae are indicative of Aspergillosis (Grocott methenamine silver staining, $\times 400)$. (C) Cholesterol clefts surrounded by fibrosis and granulomatous inflammation cells, foamy histiocytes (H\&E staining, $\times 400)$.

Table 1. Summary of epidemiologic and clinical features of fungal balls in the middle ear cavity reported in the literature so far, including the current case

\begin{tabular}{|c|c|c|c|c|c|c|c|c|}
\hline $\begin{array}{c}\text { Case } \\
\text { number }\end{array}$ & $\begin{array}{l}\text { Age } \\
\text { (years) }\end{array}$ & Sex & Symptom & Side & Past history & $\begin{array}{c}\text { Fungal } \\
\text { infection in } \\
\text { other sites }\end{array}$ & Treatment & Follow-up \\
\hline \multirow[t]{2}{*}{1} & 30 & Male & Otorrhea & Rt & No & No & Surgery & 4 month \\
\hline & & & Hearing impairment & & & & & \\
\hline 2 & 56 & Male & Hearing impairment & Rt & $\begin{array}{l}\text { Tympanoplasty } \\
\text { (21 years earlier) }\end{array}$ & No & Surgery & 2 year \\
\hline $\begin{array}{l}3 \text { (Current } \\
\text { case) }\end{array}$ & 62 & Male & $\begin{array}{c}\text { Otorrhea } \\
\text { Hearing impairment }\end{array}$ & R† & COPD & No & Surgery & 1 year \\
\hline
\end{tabular}

COPD: chronic obstructive pulmonary disease, Rt: right 
be the source of the CG [9]. The longitudinal relationship between simultaneously occurring CG and fungal balls cannot be clarified, despite similar etiologic mechanisms, such as obstruction of drainage, impaired ventilation, and hemorrhage.

As discussed, several hypotheses have been proposed to explain the pathogenesis of fungal ball formation in the middle ear. First, the chronicity of the discharge plays an important role as humid conditions in the ear alter the $\mathrm{pH}$. Second, prolonged use of topical broad-spectrum antibiotics suppresses the normal bacterial flora and increases susceptibility to opportunistic fungal overgrowth. Lastly, the mechanism may involve obstruction of ventilation and impaired drainage. As far as we know, there have been two cases of fungal balls in the middle ear cavity reported worldwide (Table 1) [10,11]. Unlike our case, there was no obstruction of the pharyngotympanic tube in previously reported cases. However, one case report described retraction of the tympanic membrane and fluid collection, which could obstruct ventilation and drainage. A humid condition of the middle ear cavity was observed in all patients, including the present case. However, use of topical broadspectrum antibiotics was not reported in previous cases. While the simultaneous occurrence of CG and fungal ball has not yet been reported, the condition could be attributed to obstruction of ventilation, impaired drainage, and hemorrhage. It is noteworthy that CT scans of fungal balls in other regions appear opacified with hyperdense areas. However, the reported cases, including ours, did not reveal any opacification or hyperdense lesions. There has been no evidence of relapse over a one-year follow-up period.

In our view, fungal ball in the middle ear should be discriminated in the presence of CG in patients with chronic otitis media, even though there is no evidence of otomcycosis. Moreover, the possibility should not be overlooked if the patient has received topical antibiotics for a prolonged period. Our inference is supported by the fact that both CG and fungal ball are well- formed in a humid environment.

\section{Acknowledgments}

This paper was supported by Fund of Biomedical Research Insti- tute, Chonbuk National University Hospital.

\section{Conflicts of interest}

The authors have no financial conflicts of interest.

\section{Author Contributions}

Conceptualization: Eun Jung Lee. Data curation: Su Geun Kim. Formal analysis: Su Geun Kim. Investigation: Su Geun Kim, Ji Seob Yoo, and Cha Dong Yeo. Visualization: Su Geun Kim. Writingoriginal draft: Su Geun Kim. Writing — review \& editing: Eun Jung Lee.

\section{ORCID iDs}

Su Geun Kim

Eun Jung Lee

Ji Seob Yoo

Cha Dong Yeo

https://orcid.org/0000-0002-5791-7544

https://orcid.org/0000-0001-6958-0312

https://orcid.org/0000-0002-1155-3335

https://orcid.org/0000-0002-5802-6444

\section{REFERENCES}

1) Evans EGV, Gentles JC. Essentials of medical mycology. 1st ed. Edinburgh, England: Churchill Livingstone;1985. p.86-7.

2) Dhingra R, Monga S, Kaur M, Brar SRS, Rupali, Arora H. Role of fungal infections in CSOM: prospective study. Indian J Basic Appl Med Res 2014;3:598-608

3) Rippon JW. Medical mycology: the pathogenic fungi and the pathogenic actinomycetes. 2nd ed. Philadelphia, USA: WB Saunders; 1982. p.491.

4) Kunelskaya VY. [Significance of fungal flora in chronic suppurative otitis media]. Vestn Otorinolaringol 1969;31:28-32.

5) Gherini SG, Brackmann DE, Lo WW, Solti-Bohman LG. Cholesterol granuloma of the petrous apex. Laryngoscope 1985;95:65964.

6) Jackler RK, Cho M. A new theory to explain the genesis of petrous apex cholesterol granuloma. Otol Neurotol 2003;24:96-106.

7) Sarioglu S, Pabuççuoglu U, Arzu Topal N. Cholesterol granuloma and aspergilloma of the maxillary sinus. Eur Arch Otorhinolaryngol 2001;258:74-6.

8) Kang H, Kim JK, Kim Y. Association of cholesterol granuloma and aspergillosis in the sphenoid sinus. Korean J Radiol 2008;9 Suppl: S30-3.

9) Carlile MJ, Watkinson SC, Gooday GW. The fungi. 1st ed. London, England: Academic Press;1994. p.86.

10) Kim JH, Song CE, Jun BC, Lee DH. A case of fungal ball of Aspergillus in chronic otitis media. Korean J Otolaryngol 2006;49: 651-4.

11) Supiyaphun P, Sampatanukul P, Sukumalpaiboon P. Benign Aspergillus colonization (Aspergilloma) in the middle ear. Otolaryngol Head Neck Surg 2001;125:281-2. 